\title{
Indications for surgical reintervention following reverse shoulder arthroplasty: a retrospective audit from 2006 to 2015
}

\author{
Du Plessis $\mathrm{CP}^{1}$, Koch O², Le Roux $\mathrm{TLB}^{3}$, Janse van Rensburg $\mathrm{C}^{4}$ \\ $\mathrm{MBChB}($ Stell); Orthopaedic Registrar, University of Pretoria \\ MBChB(Pret), FCS Orth(SA), MMed(Ortho)(Pret); Orthopaedic Surgeon, Department of Orthopaedics, 1 Military Hospital, University of Pretoria \\ MBChB(Pret), FCS Orth(SA), MMed(Ortho)(Pret); Professor and Head of Department, Department of Orthopaedics, 1 Military Hospital, University of \\ Pretoria \\ 4 BSc Actuarial \& Financial Mathematics(Pret), BSc(Hons) Mathematical Statistics(Pret); Biostatistician South African Medical Research Council
}

Corresponding author: Dr CP du Plessis, Department of Orthopaedic Surgery, Steve Biko Academic Hospital, Faculty of Health Sciences, University of Pretoria, Private Bag x 323, Pretoria, 0020; Email: flipduplessis00@gmail.com; Tel: +27 012354 2851; Fax: +27 0123542821

\begin{abstract}
Background: Reverse shoulder arthroplasty (RSA) has increased in popularity and its indications have subsequently been expanded. With its increased use, the complication rates have also increased. Complications requiring additional surgeries have the highest morbidity and cost. The aim of this study was to determine the indications for additional surgery following RSA.

Methods: All the surgical and clinical notes of patients treated with an RSA at our institution over a nine-year period were retrospectively reviewed. Sixty-seven RSAs met the inclusion criteria and their records were reviewed to assess their indication for surgery, complications, as well as microbiology results if infection was present.

Results: Surgical reintervention was required in 16 (23.9\%) RSAs. The prevalence was lowest in rotator cuff arthropathy and glenohumeral arthritis (nine RSAs or 18.4\%), followed by failed hemi- or total shoulder arthroplasty (four RSAs or 36.4\%) and highest if performed for uncommon conditions (two RSAs or 66.7\%). Instability was an early complication, occurring in $10.7 \%$ of cases and accounting for $37.8 \%$ of all reinterventions. Infection was a late complication, occurring in $6.0 \%$ of cases and accounting for $48.6 \%$ of all reinterventions. The most common organisms identified were Staphylococcus epididermidis $(n=4)$, Escherichia coli $(n=3)$, Staphylococcus aureus ( $\mathrm{n}=2$ ) and Klebsiella pneumonia $(\mathrm{n}=2)$.

Conclusions: RSA has the most reliable outcomes if performed for rotator cuff arthropathy and glenohumeral osteoarthritis. Instability and infection are the most common indications for surgical reintervention, and once present, often require repeated surgeries to be successfully treated. These complications should be avoided, as they are major contributors to morbidity and cost.
\end{abstract}

Level of evidence: Level 4

Key words: reverse shoulder arthroplasty, reverse shoulder replacement, complication, reoperation, revision, reintervention

Citation: Du Plessis CP, Koch O, Le Roux TLB, Janse van Rensburg C. Indications for surgical reintervention following reverse shoulder arthroplasty: a retrospective audit from 2006 to 2015. SA Orthop J 2017;16(4):28-32. http://dx.doi.org/10.17159/2309-8309/2017/v16n4a4

Editor: Prof Anton Schepers, University of the Witwatersrand

Received: April 2017 Accepted: July 2017 Published: November 2017

Copyright: @ 2017 Du Plessis CP, et al. This is an open-access article distributed under the terms of the Creative Commons Attribution Licence, which permits unrestricted use, distribution and reproduction in any medium, provided the original author and source are credited.

Funding: No funding was received with respect to this article.

Conflict of interest: Drs Du Plessis, Koch and Prof Le Roux declare that they have no conflict of interest. No benefits of any form have or will be received from any commercial party related directly or indirectly to the subject of this article. 


\section{Introduction}

The French surgeon, Dr Jules-Émile Péan, performed the first shoulder arthroplasty in 1893. Although this prosthesis lasted for only two years before it had to be removed, this was a remarkable accomplishment for the time when it was performed. Almost 60 years passed before Neer developed an anatomic prosthesis for hemiarthroplasty of the shoulder in the 1950s.

The next development after anatomic shoulder arthroplasties was the non-anatomic reverse shoulder arthroplasty (RSA). ${ }^{2,3}$ In this design the glenoid component is spherical and articulates with the concave humeral component. The result is a new biomechanical environment in which the deltoid muscle can compensate for deficient rotator cuff muscles. ${ }^{3}$

Since its approval for use in the United States of America in 2003, the use of RSA has sharply increased. 4,5 The 2.5-fold increase in the use of shoulder arthroplasties from 2000 to 2008 is largely attributed to the introduction of RSA. ${ }^{5}$ Villacis et al. reported a $70 \%$ increase in the use of RSA in the American state of California between 2011 and 2013. ${ }^{6}$

The initial indication for the use of RSA was for elderly patients with severe rotator cuff arthropathy. 2,5 However, with increased use, the indications for the procedure have been expanded, leading to younger patients also being offered RSA., 4,6-8

This increased use has been associated with an increased complication rate, which is reported to be $4.8-68 \%{ }^{6}$ The high variability in complication rate can be explained by the inclusion of complications in some studies that are not clinically relevant. Zumstein et al. found that complications leading to revision surgery had a negative effect on the final functional outcome of patients. They defined reintervention as any subsequent surgery on the operated shoulder. This was further divided into reoperations ('interventions requiring any return to the operating room for any reason relating to the shoulder, without altering or replacing any of the components') and revisions ('surgeries with total or partial exchange or removal of the components'). ${ }^{9}$

In the literature currently, reintervention rates range between 5.3 and $15 \%{ }^{7,9,10}$ The indications for reinterventions have been investigated by previous authors, but many of the published studies either have a small sample size, ${ }^{10,11}$ short follow-up ${ }^{7,11}$ or incomplete reporting on their indications for reintervention. $6,11,12$ Two notable exceptions to the above are a systematic review by Zumstein et al. ${ }^{9}$ and a review article by Boileau. ${ }^{13}$

These patients are also typically elderly with multiple co-morbidities that make them unsuitable for repeated high-risk anaesthetics (beach-chair position). ${ }^{6}$ Repeated surgical interventions also place a significant monetary burden on the health care system as well as individuals.

The purpose of this study was to determine the prevalence of surgical reintervention in RSA, as well as the specific indications for reintervention.

\section{Material and methods}

The records of all patients who had undergone an RSA at our unit between November 2006 and September 2015 were retrospectively reviewed. Patients were excluded if their RSA was not performed at our unit or if they did not have a minimum follow-up of one year.

The following data was recorded:

- The indication for the RSA and the number of shoulders that had reinterventions (Table I)

- The indications for surgical reintervention; the number of reinterventions performed; whether it was a reoperation or revision (Figures 1 and 2)

- The interval between reintervention and initial RSA

- In cases of infection, the microbiology results were retrieved to determine the involved organisms (Table I/)
Initially the prosthesis was implanted through a deltopectoral approach, but the approach was gradually changed to superolateral due to its improved stability according to the literature.,13 Subscapularis was not routinely repaired and no patients had latissimus dorsi transfers. Patients were operated in the beach-chair position under general anaesthesia, augmented with a regional block if deemed fit by the anaesthesiologist. Wounds were closed over a Porto-Vac suction drain and the shoulder put in an arm immobiliser post-operatively. Multiple fluid samples were taken if infection was suspected and sent in aerobic and anaerobic blood culture bottles for processing.

\section{Statistical analysis}

The descriptive statistics mean, standard deviation, median and range with 95\% confidence intervals for continuous variables and proportions with 95\% confidence intervals for categorical variables were calculated. The proportions were used to determine the prevalence of surgical reintervention in RSA. The t-test, chi-square and Fisher exact tests were used to test for any associations between categorical variables. Tests were evaluated at $5 \%$ level of significance. All analysis was done using STATA 14

\section{Results}

There were 71 patients that underwent a total of 77 RSAs. Sixty-two patients with a total of 67 RSAs were enrolled in the study. The following patients were excluded: four patients that died of unrelated causes before a one-year follow-up, three patients because of a short follow-up and two patients due to incomplete records. One patient with bilateral replacements had to be excluded.

Twenty-five (37.3\%) RSAs were performed on the left side and $42(62.7 \%)$ on the right side. Twenty-six prostheses (38.8\%) were implanted through a deltopectoral approach and 41 (61.2\%) through a superolateral approach. Forty-one (61.2\%) replacements were for females and 26 (38.8\%) for males with an average age of 68.7 years (55-85 years). The mean follow-up was 50.8 months (12-127 months).

The most common indication for an RSA was rotator cuff arthropathy $(n=41,61.2 \%)$. A total of $16(23.8 \%)$ replacements required a reintervention (Table $)$. Seven (10.4\%) of these had post-operative instability, four (6.0\%) infection and five $(7.5 \%)$ other less common complications (Figure 1). When comparing age $(p=0.644)$, sex $(p=0.292)$ and operated side $(p=0.986)$, there was no statistically significant difference between patients needing a reintervention and those that did not.

Table I: Primary indications for RSA showing the number of patients that had surgical reinterventions

\begin{tabular}{|l|c|c|}
\hline Primary indication & Number of RSA & $\begin{array}{c}\text { RSA that had } \\
\text { reinterventions }\end{array}$ \\
\hline $\begin{array}{l}\text { Rotator cuff arthropathy } \\
\text { Failed hemi shoulder } \\
\text { arthroplasty }\end{array}$ & $41(61.2 \%)$ & $9(22.0 \%)$ \\
\hline $\begin{array}{l}\text { Glenohumeral osteoarthritis } \\
\text { Failed ORIF of proximal } \\
\text { humeral fractures }\end{array}$ & $8(11.9 \%)$ & $3(33.3 \%)$ \\
\hline $\begin{array}{l}\text { Miscellaneous } \\
\text { Failed total shoulder } \\
\text { arthroplasty }\end{array}$ & $4(6.0 \%)$ & $1(25.0 \%)$ \\
\hline Total & $3(4.5 \%)$ & $2(66.7 \%)$ \\
\hline
\end{tabular}

The miscellaneous group included an ancient shoulder dislocation, chondrosarcoma and metastatic breast cancer. ORIF = Open Reduction and Internal Fixation. RSA $=$ Reverse Shoulder Arthroplasty 




Figure 1. The indications for initial reintervention after RSA

${ }^{*}$ Removal of a prominent screw used to ORIF a previous os acromiale now irritating the deltoid muscle and repair of a deltoid muscle tear

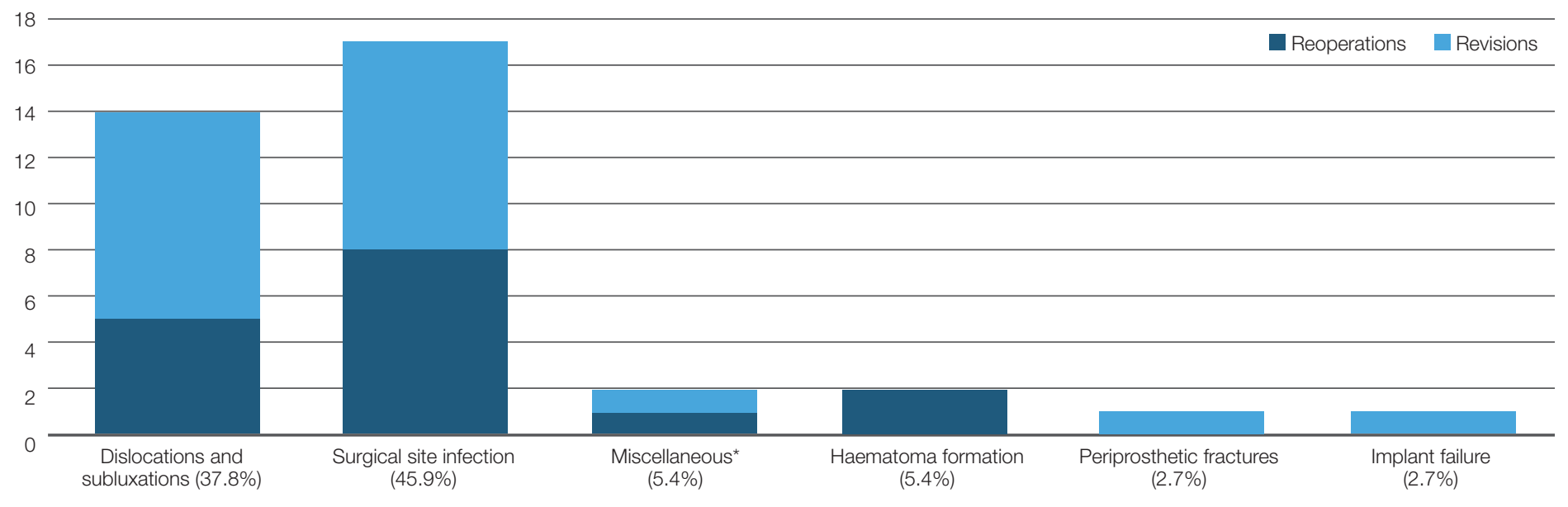

Figure 2. Total number of reinterventions required

${ }^{*}$ Removal of a prominent screw used to ORIF a previous os acromiale now irritating the deltoid muscle and repair of a deltoid muscle tear

Table II: Epidemiology of the infective organisms

\begin{tabular}{|l|c|c|c|}
\hline Pathogen & $\begin{array}{c}\text { No times } \\
\text { identified }\end{array}$ & $\begin{array}{c}\text { No times } \\
\text { identified (\%) }\end{array}$ & $\begin{array}{c}\text { Patients } \\
\text { identified (\%) }\end{array}$ \\
\hline Staphylococcus epididermidis & 4 & 23.5 & 50.0 \\
\hline Escherichia coli & 3 & 17.6 & 37.5 \\
\hline Staphylococcus aureus & 2 & 11.8 & 25.0 \\
\hline Klebsiella pneumonia & 2 & 11.8 & 25.0 \\
\hline Candida albicans & 1 & 5.9 & 12.5 \\
\hline Streptococcus viridans & 1 & 5.9 & 12.5 \\
\hline Stenotrophomonas maltophilia & 1 & 5.9 & 12.5 \\
\hline Pseudomonas aeruginosa & 1 & 5.9 & 12.5 \\
\hline Enterobacter cloacae & 1 & 5.9 & 12.5 \\
\hline Enterococcus faecium & 1 & 5.9 & 12.5 \\
\hline
\end{tabular}

A total of 37 reinterventions were performed. Seventeen $(45.9 \%)$ of these were for surgical-site infection and 14 (37.8\%) for instability (Figure 2). The reinterventions were not adjusted for the number of times a specific shoulder had to go back to theatre.

Surgical reintervention was performed at a mean of 14.8 months (0-67 months). Six of the nine reinterventions that were performed within three months of surgery were for instability. Surgical-site infection $(n=2)$ and haematoma formation $(n=1)$ also occurred within three months of RSA. Surgical site infection $(n=2)$, deltoid problems $(n=2)$, periprosthetic fractures $(n=1)$, instability $(n=1)$ and implant failure $(n=1)$ occurred after three months.

Microscopy and culture results revealed a polymicrobial infection in four shoulders and monomicrobial infection in three shoulders. In one case no organism was identified, but this patient was on suppressive antibiotics for five weeks before revision. Escherichia coli, Klebsiella pneumonia and Pseudomonas aeruginosa were the only organisms that occurred in isolation. Staphylococcus epididermidis $(n=4)$ was the most commonly isolated organism, followed by E. coli $(\mathrm{n}=3)$, Staphylococcus aureus $(\mathrm{n}=2)$, K. pneumonia $(n=2)$ and a number of other pathogens (Table II).

More than one organism was identified in five patients (62.5\%) 


\section{Discussion}

RSA has gained in popularity over the last two decades. While it was originally indicated for patients with rotator cuff arthropathy, the indications have been expanded as it became more commonly used. Common indications are rotator cuff arthropathy (26.7-82.5\%), glenohumeral osteoarthritis (25.1\%), acute proximal humerus fractures (2.3-38.5\%), revisions of hemi- or total shoulder arthroplasty (2.5-27.6\%), rheumatoid arthritis (2.9-10\%), failed open reduction and internal fixation (ORIF) of proximal humerus fractures (5.2\%) and tumours (0.8\%). 4,6,7,9,10,12 Similar indications for RSA as mentioned above were found in our cohort, with the exception of rheumatoid arthritis and acute proximal humerus fractures (Table $)$.

The complication rate was lowest if the indication for RSA was rotator cuff arthropathy or glenohumeral osteoarthritis (18.4\%). When performed as a revision procedure for a failed arthroplasty, the complication rate increased to $36.4 \%$. The highest complication rate $(66.7 \%)$ occurred when RSA was performed for expanded indications, which were an ancient dislocation and tumour-related procedures (Figure 3). Zumstein et al. found that the incidence of all kinds of complications increased from $28.7 \%$ for primary RSA to $65.7 \%$ for revision cases. ${ }^{9}$ Similarly Russo et al. found an increase from $10.8 \%$ to $36.8 \% .^{12}$ This suggests that, although RSA may be a useful method to treat complex shoulder problems, it should be utilised with care in these patients as the best outcomes are seen in patients with primary shoulder pathology.

Post-operative instability was the most common complication encountered at $10.4 \%$ and accounts for $43.8 \%$ of all complications. This supports previous findings with instability rates of between 2.4 and $31 \% .{ }^{4,9,13,14}$ Villacis et al. reported an early dislocation rate (before 90 days) of $1.8 \%$ with an increase to $5.5 \%$ at two years. ${ }^{6}$

In the current study, it was found that dislocation is an early complication with $85.7 \%(6 / 7)$ of the cases presenting within three months of RSA primary surgery. At 66.7\% (6/9) it was also the most common complication occurring within three months. Although there is an association between the deltopectoral approach and instability due to subscapularis muscle violation, it could not be confirmed $(p=0.557) .4,13$ Instability was found in $7.7 \%$ of shoulders operated through a deltopectoral approach, compared to $12.2 \%$ of those approached through the more commonly used superolateral incision. Technical errors during surgery explain why instability is more frequently an early complication. These include failure to restore adequate soft-tissue tension of the deltoid, incorrect position (version) of the prosthesis, excessive medialisation or inadequate diameter of the glenosphere and not removing tissues inferior to the glenosphere that can cause mechanical impingement. Additionally, failure to recognise and restore humeral and glenoid bone loss as well as not repairing the subscapularis muscle when possible may contribute to instability. ${ }^{4,13}$ Meticulous detail to the above can help to reduce dislocation rates.

Fourteen (37.8\%) of the reinterventions occurring in seven patients were for instability, indicating that this is a recurrent problem. This supports previous findings indicating that $38-59 \%$ of RSA shoulders will remain unstable after reduction. ${ }^{13}$ The sample size was too small to determine a relationship between the indication for a RSA and instability.

Infection was the second most common complication in our cohort, initially affecting four (6.0\%) RSAs and accounting for $25.0 \%$ of the complications. A further four cases developed infection after reintervention, two of which followed revisions for instability. One patient required six reinterventions for infection. This suggests that repeated surgeries as well as instability contribute to infection. Infection required the most reinterventions (45.9\%), emphasising the difficulty in treating this complication. The post-operative infection rate is reported as $1-10 \%$ in the literature and up to $12 \%$ after revision surgery. ${ }^{4,9,13-16}$ Risk factors for post-operative infection

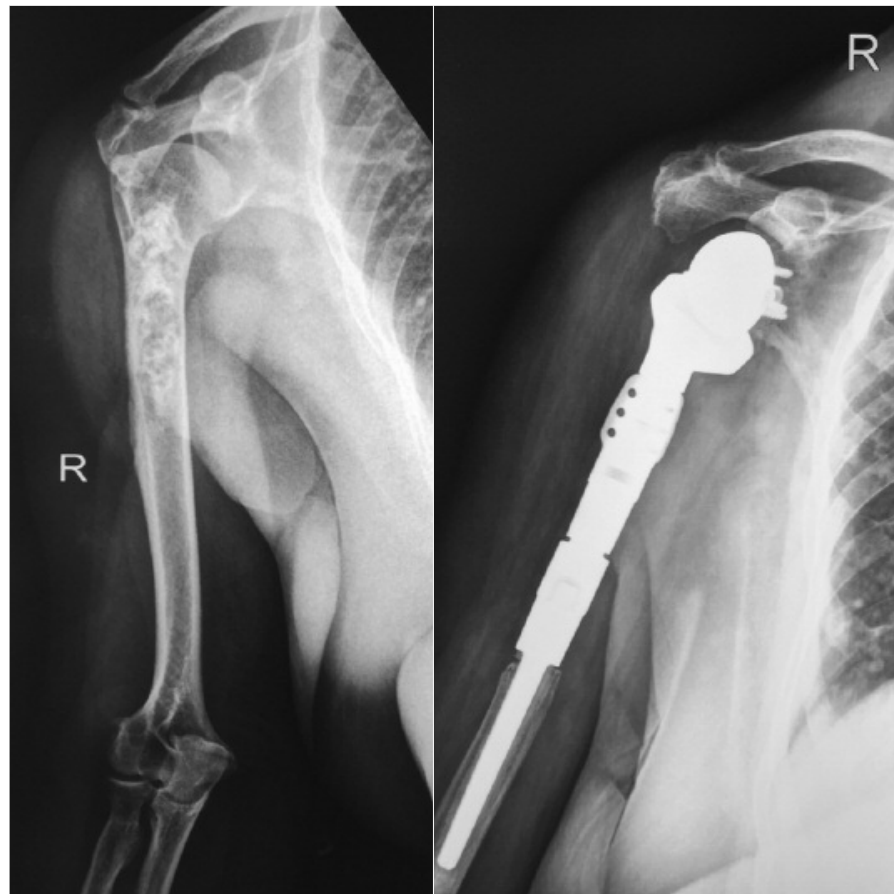

Figure 3. 70-year-old female with chondrosarcoma of her right proximal humerus successfully treated with a RSA tumour prosthesis

are prior failed arthroplasty, multiple surgeries, age less than 65 years, instability, haematoma formation and inadequate dead space management. ${ }^{4,15}$ Infection occurred at a mean of 22.1 months after RSA, suggesting it is a late complication. This is in keeping with trends seen in the literature where there is an increased incidence of infection after one year.,15,16 The late presentation may be explained by an unrecognised latent low-grade infection sustained during the primary or revision surgery. ${ }^{4}$

The organism most commonly identified was $S$. epididermidis followed by E. coli, S. aureus and K. pneumonia. The most frequently identified bacteria causing infection after RSA in two recent studies were Propionibacterium acnes, S. epididermidis and $S$. aureus. ${ }^{15,16}$ We encountered no cases of $P$. acnes. This may be due to our local pathogen profile or the prolonged culture that is needed to detect $P$. acnes. ${ }^{4,15}$

Infection can be avoided by pre-operative chlorhexidine body washes, administrating adequate prophylactic antibiotics within one hour of surgery, using antibiotic-impregnated cement, atraumatic soft-tissue handling and by placing drains to manage the dead space left by the removal of the rotator cuff. ${ }^{4}$ Despite all these precautions it must be realised that the elderly population are often poor hosts due to their medical co-morbidities, nutritional status and often very advanced age. This predisposes them to a higher risk of infection. ${ }^{17}$

Instability and infection combined accounted for 11 (68.8\%) of the patients that required reintervention and 31 (83.8\%) of all the reinterventions that were performed, often requiring multiple procedures in the same patient. Periprosthetic fractures, implant failure and haematoma formation that reportedly accounts for $1.4-4.0 \%, 1.8-29 \%$ and $2-3 \%$ of complications respectively, were rare in the sample group..$^{9,12-14}$ Deltoid problems consisted of a prominent screw after a previous os acromiale ORIF irritating the deltoid muscle, necessitating removal, and a tear in the deltoid muscle requiring repair due to persistent pain. Deltoid injury is a rare complication, accounting for $0.9 \%$ of all complications. ${ }^{13}$

The pricing of prostheses is one of the big cost drivers in arthroplasty surgery. Revision surgery requires component exchange, and accounted for 21 (56.8\%) of the reinterventions performed, indicating the monetary burden on the health care system. 
The inherent weaknesses of a retrospective study are acknowledged. However, comprehensive follow-up and recordkeeping with electronically captured theatre and outpatient notes were done. Patients excluded from the study were few and mainly due to unrelated death before minimum follow-up. Our large scope of practice helped minimise selection bias. The relatively small sample size made the assessment of rare complications and comparisons between different groups difficult. The average follow-up was long at 50.8 months, even though the minimum follow-up for inclusion was 12 months.

\section{Conclusion}

Although there has been a tendency to use RSA to treat complex shoulder problems, our data suggests that the outcomes are better when used for primary shoulder pathology such as rotator cuff arthropathy and glenohumeral arthritis. Care should be taken when dealing with other indications, especially if there are other treatment options available. The reintervention rate doubles when treating failed arthroplasty with RSA and increases almost four times when it is used to treat uncommon conditions like ancient dislocations and tumours. Instability is the most common complication and occurs early post-operatively. It is often a recurrent problem.

Sepsis is the second most common cause for reintervention but the most difficult to treat, requiring repeated reinterventions. It is a problem that often develops after repeated surgeries, more commonly after reintervention for instability.

Once a complication arises that requires reintervention, multiple procedures are often needed, adding to patient morbidity and health care cost.

\section{Compliance with ethics guidelines}

The ethical committees of our institution as well as the hospital where the research was performed gave approval before the research commenced.

\section{References}

1. Mehmood A, Everett W. Shoulder replacements: a review. Br J Hosp Med (Lond) 2016;77:84-89.

2. Grammont $P$, Trouilloud $P$, Laffay $J$ Deries $X$. Etude et realisation d'une nouvelle prothese d'epaule. Rheumatologie 1987;39:407-18.

3. Boileau P, Watkinson DJ, Hatzidakis AM, Balg F. Grammont reverse prosthesis: design, rationale, and biomechanics. J Shoulder Elbow Surg. 2005;14:147S-161S.

4. Cheung E, Willis M, Walker M, Clark R, Frankle MA. Complications in reverse total shoulder arthroplasty. J Am Acad Orthop Surg. 2011; 19:439-49.

5. Kim SH, Wise BL, Zhang Y, Szabo RM. Increasing incidence of shoulder arthroplasty in the United States. J Bone Joint Surg Am. 2011;93: 2249-54.

6. Villacis D, Sivasundaram L, Pannell WC, Heckmann N, Omid R, Hatch GF, 3rd. Complication rate and implant survival for reverse shoulder arthroplasty versus total shoulder arthroplasty: results during the initial 2 years. J Shoulder Elbow Surg. 2016 Jun;25(6):927-35

7. Groh GI, Groh GM. Complications rates, reoperation rates, and the learning curve in reverse shoulder arthroplasty. J Shoulder Elbow Surg. 2014;23:388-94.

8. Sershon RA, Van Thiel GS, Lin EC, et al. Clinical outcomes of reverse total shoulder arthroplasty in patients aged younger than 60 years. $J$ Shoulder Elbow Surg. 2014;23:395-400.

9. Zumstein MA, Pinedo M, Old J, Boileau P. Problems, complications, reoperations, and revisions in reverse total shoulder arthroplasty: a systematic review. J Shoulder Elbow Surg. 2011;20:146-57.

10. Guery J, Favard L, Sirveaux F, Oudet D, Mole D, Walch G. Reverse total shoulder arthroplasty. Survivorship analysis of eighty replacements followed for five to ten years. J Bone Joint Surg Am. 2006;88:1742-47.

11. Wierks C, Skolasky RL, Ji JH, McFarland EG. Reverse total shoulder replacement: intraoperative and early postoperative complications. Clin Orthop Relat Res. 2009;467:225-34.
12. Russo R, Rotonda GD, Ciccarelli M, Cautiero F. Analysis of complications of reverse total shoulder arthroplasty. Joints 2015;3:62-66.

13. Boileau P. Complications and revision of reverse total shoulder arthroplasty. Orthop Traumatol Surg Res. 2016;102:S33-43.

14. Bohsali KI, Bois AJ, Wirth MA. Complications of shoulder arthroplasty. J Bone Joint Surg Am. 2017;99:256-69.

15. Morris BJ, O'Connor DP, Torres D, Elkousy HA, Gartsman GM, Edwards TB. Risk factors for periprosthetic infection after reverse shoulder arthroplasty. J Shoulder Elbow Surg. 2015;24:161-66.

16. Jacquot A, Sirveaux F, Roche O, Favard L, Clavert P, Mole D. Surgical management of the infected reversed shoulder arthroplasty: a French multicenter study of reoperation in 32 patients. J Shoulder Elbow Surg. 2015;24:1713-22.

17. Wester AL, Dunlop O, Melby KK, Dahel UR, Bruun T. Age-related differences in symptoms, diagnosis and prognosis of bacteremia. BMC Infectious Diseases. 2013;13:346-58. 